\title{
Design and Implementation of WMMP in Medical Application
}

\author{
Wei Zhao \\ Industrial Control Networks and Systems Department \\ Shenyang Institute of Automation \\ Shenyang, China \\ zwneu@sia.cn
}

\author{
Gang Shi \\ Industrial Control Networks and Systems Department \\ Shenyang Institute of Automation \\ Shenyang, China \\ sg0105@sia.cn
}

\begin{abstract}
As an important part of the next generation network, Internet of things is affecting every aspect of our lives. Now an accepted the conclusion is that M2M technology is the specific form of concrete implementation of Internet of things in current conditions. In order to realize the machine to machine communication, the communication protocol between the terminal and platform is necessary. As the leader of IoT in China, China Mobile has published Wireless Machine Management Protocol (or WMMP, in short). WMMP is a set of very complete M2M protocol specification and it is easy to program on all kinds of embedded device. The critical function of WMMP is a set of mechanism which manages all connecting devices. The management mechanism includes the following parts: device register, login, configuration parameter setting, configuration parameter getting, configuration parameter report, remote control, application data transmitting and firmware updating. In this paper, the related knowledge of WMMP is introduced and the how to applied it in a medical device is also discussed. Practice has proved that WMMP can not only satisfy the requirement of the M2M communications, but also has good expansibility and broad applicability.
\end{abstract}

Keywords- Internet of things; M2M; WMMP;

\section{INTRODUCTION}

Technologies change our life. Out of many emerging technologies, Internet of Things (IoT), also known as machine-to-machine (M2M) (where smart devices that collect data, relay information to one another, process the information collaboratively, and take action automatically) is a new paradigm offering both challenges and opportunities, as in [1].

Internet of things is not a new concept, and the general pattern of it is: data acquisition, transmission, application platform to form network. As at the beginning of the Internet is made up of small local area network (LAN), a common consensus is that the existing M2M applications is the composition of the Internet of things.

On the other hand, medical wisdom, as an emerging discipline, is a blend of life science and information technology. The key technology of intelligent medical is an important part of modern medicine and communication technology. Medical wisdom through building in electronic health records as the center of regional health information platform, the use of the Internet of things technology, realizes the patients and medical staff, medical institutions, medical equipment, gradually achieve the comprehensive information. At present, some of the medical model mentioned frequently, such as wireless medical, mobile medical and physical health, etc, the core characteristics of the concept of the above are all belong to the category of medical wisdom. In this article, the community or family self-help health care service system, which combining the physiological signal detection, and the Internet of things technology, belongs to a kind of realization forms of the medical wisdom.

\section{SOME ASSOCIATED CONCEPTS}

\section{A. Internet of things}

The concept of Internet of things, was first put forward in 1999, and its main idea can be described as follows: through information sensing devices, such as radio frequency identification (RFID), infrared sensors, global positioning system, laser scanner, gas sensors etc, according to the contract agreement, connecting any item with the Internet, proceeding information exchange and communication, in order to realize intelligent identification, location, tracking and monitoring and management of a network. In short, the Internet of things is "everything connected to the Internet".

According to the international telecommunication union (ITU), the definition of the Internet of things mainly solve the items with the items (Thing to Thing, T2T), people and objects (the Human to Thing, H2T) and people (the Human to Human, $\mathrm{H} 2 \mathrm{H}$ ) between the interconnection. But unlike traditional Internet, H2T refers to use the connection between the general equipment and goods, to make things more simplified, and the $\mathrm{H} 2 \mathrm{H}$ refers to is not dependent on the PC for the interconnection between people. Because the Internet has no connection to any items into consideration, so we use the Internet to solve this problem in the traditional sense. As the name suggested, the Internet of things is the network connection objects. Many scholars often introduce a concept of M2M to discuss the Internet of things, can explain become people to people, Man to Man, and Man to the Machine (Man) to the Machine, Machine to Machine (the Machine to Machine). In essence, the interaction between people and machines, machines and machines, mostly in order to realize the information interaction between people, as in [4] and [5].

\section{B. $M 2 M$}

M2M technology belongs to the category of Internet of things, but that is not the same as the Internet of things. It is 
through the communication between machines, machine control communications, mobile communications, interactive communication. M2M is a combination of data collection, data transmission, remote control, terminal equipment, machinery, transmission network, application processing platform and integrities all above into one on the logical entity. M2M technology makes the machine equipment networking communication ability, and makes it happen that man communicate with machine and machine communicate with machine without thinking of the limit of time and space, as in [2] and [3].

The vertical system of IoT framework is a structure of three layers: collection and initial information perception layer, information data reliable transmission network layer, and information remote processing decisions application layer. In the integrated network system, we can simplify the core essence of which is to transmit data information from one end of the equipment to the other end of the machinery and equipment, including machines. The interconnection between the communication, that is, M2M technology. So we can consider that M2M is a specific instance of the Internet of things. When so many independent local M2M applications realize large-scale, heterogeneous network tile instrumentation, independent M2M system through the connectivity between network communication intelligent fusion, this is the true sense of the Internet of things, therefore, M2M is the concrete implementation and composition for the implementation of Internet of things.

\section{RELATED WORK}

\section{A. System Carrier}

The carrier of M2M communication is a set of device which is designed to meet the needs of medical test in some grassroots situation, such as village, community and force.

Integrated health tester is designed for grassroots medical applications ,so it has the characteristics of small, multifunction and easy to carry. Six basic physiological parameters detection can be done through it, including ecg, body temperature, blood pressure, pulse frequency, blood oxygen saturation and respiratory rate. It can also proceed other biochemical parameters detection for medical applications, such as dry blood biochemical analysis, hemoglobin analysis, cross matching test, clotting factors test, ion detection, blood-gas analysis.

In order to be applied in the bad environment, the reliability of integrated health tester is taken into account in the beginning of design. The military level of motherboard with SSD and low temperature type battery energy are used and the main circuit boards are protected by special paint which can resist of moisture proof, smoke, and mould.

Considering the actual application scenario, the integration of health tester has carried on the corresponding design in some aspects, such as intelligent, informatization and networking in order to solve many problems in the remote medical treatment. Medical service is realized by using intelligent software, new graphics technology to realize data visualization, "fool" mode of operation, friendly interface, and simple operation process. According to the test results, medical reminders, drugs recommended and rehabilitation programme can be given automatically. On the other hand, local health files can be established and test results can be filled and stored in files automatically. The identity of the tested object information can be directly read by means of documents scanning software interface. Through the embedded micro thermal printer, the test result can be printed directly. The technology of rapid physiological indexes detection based on body sensor network can reduce the psychological and physiological burden of critically ill patients. At the same time, using the mechanism of $\mathrm{M} 2 \mathrm{M}$, test results can be transmission to the server platform via mobile 3G/2G network. Doctors can offer remote diagnosis and monitoring guidance to the medical staff through service system platform.

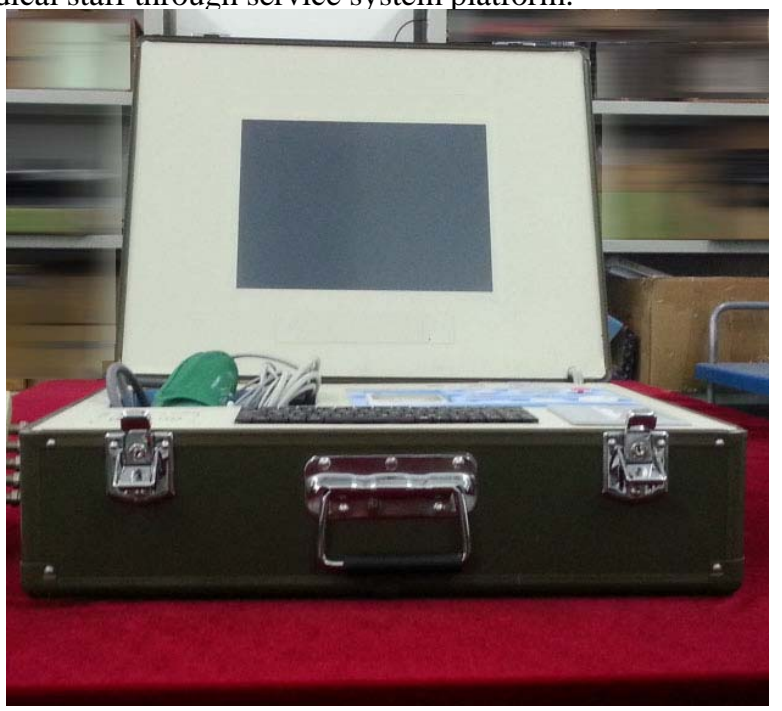

Figure 1. The appearance of integrated health tester

\section{B. M2M Communication Protocal}

WMMP (Wireless M2M Protocol) Protocol is established by China Mobile for M2M business between M2M terminal and M2M terminal, M2M terminal and M2M platform, M2M platform and M2M application platform for data communication in data processing layer. The designed system is shown as follow.

WMMP agreement by two parts: M2M platform and M2M terminal interface (WMMP - T) and M2M platform and M2M applications interface protocol (WMMP - A). WMMP - T agreement data communication between M2M terminal and M2M platform. The data transmitting from one M2M terminal to the other M2M terminal and the implementation of the end-to-end routing are also defined by WMMP-T. WMMP -A agreement data communication between M2M platform and M2M application. The data transmitting from one M2M terminal to another M2M application and the implementation of the end-to-end routing are also defined by WMMP-A.

WMMP protocol is the core of the extensible protocol stack and message structure, and in its outer layer is the core 
of access by WMMP agreement has nothing to do with communication mechanism and security mechanism. On this basis, from inside out, in turn, for WMMP M2M terminal management functions and WMMP M2M applications extend the functionality.

WMMP have nothing to do with the specific business applications, only to equipment state of registered login, query, parameter setting, remote control, firmware updates, and other functions, these functions are devices or terminals in the actual application of the universal demand, in addition to business behavior, are accomplished by WMMP TRANSPARENT_DATA service. Of equipment state parameter, for example, the query, including the GPRS access point name, M2M platform IP address and port number, packet retransmission time and times, etc., and for a specific AD channel measurements, do not belong to the scope of the query WMMP agreement, should be determined by the application data report (TRANSPARENT_DATA services).

\section{The Structure of WMMP}

WMMP protocol based on TCP/IP or UDP/IP protocol and its protocol stack structure as shown in figure 2.3

Under the condition of poor network quality, it is recommended that the priority in use the UDP protocol. Such as when using GPRS as access, the UDP protocol is recommended as a transport layer protocol, this is due to the GPRS network bandwidth is narrow, longer delay, not suitable for using TCP protocol to communicate. Using UDP communication, can improve the transmission efficiency, reduce the data traffic, save network bandwidth resources. UDP is a connectionless, message-oriented data transmission protocol, compared with TCP, it has two fatal weakness: one is that there is no confirmation mechanism, data packets get lost; Second, the packet disorder. Therefore, M2M data through the UDP communication process of the upper application layer WMMP protocol implementation similar to TCP package confirmation and retransmission mechanism, so as to improve the efficiency and reliability of communication. According to practical experience, the use of UDP transmission, packet loss rate can be controlled under $1 \%$, can meet the needs of the M2M applications, as in [6].

\section{Packet Classification}

WMMP message allocation according to the message whether to adopt safety mechanism, can be divided into four categories: common message, access security verification message, encrypted message and fully encrypted message.

1) Common message. WMMP message only the contents of the packet header and submitted to the literary style in the body, do not take any security mechanism, transmitted in clear text mode.

2) Access security verification message. Outside the packet header and content of the body, at the end of the report style carries the WMMP message for access to the validation of the body, in clear text mode.
3) Encrypted message. On the content of one or a few the TLV individually encrypted, outside the packet header and content of the body, at the end of the report style carries the body WMMP message for access security verification.

4) Fully encrypted message. In addition to the packet header, the whole content is encrypted, and at the end of the report style carries the body WMMP message for access security verification.

\section{E. Communication Mode}

Logical connection is adopted in WMMP communication. Logical connection refers to the process that the M2M terminals and M2M platform finish a full message interaction together. M2M terminal sends the login in request message to M2M platform, if this login-in request is adopted by M2M platform and recognized as valid, the reply message will be sent to the M2M terminal and this replay can be seen as the start of communication both sides. The end of communication is realized by the following pattern: the M2M terminal or the M2M platform launches out and the other side sent out a reply. A special situation is also recognized as the end of communication when a logical connection timeout happens.

Because of using UDP manner, packet loss or timeout is inevitable between M2M terminals and M2M platform communication. Therefore, in order to guarantee the reliable transmission of data, use the packet retransmission mechanism is used in WMMP protocol. Based on the frequency of packet loss and overtime, M2M terminals and M2M platform for communication between anomaly can be divided into two cases: occasional exception and communication failure.

1) Occasional exception: Occasional exception refers to packet loss which is sudden or infrequent between M2M terminals and M2M platform. In occasional exceptions, the timeout retransmission mechanism is applied to solve this problem .

2) Communication failure: When packets loss constantly or the ratio of overtime is higher, the M2M terminals and M2M platform are in a state of communication failure. The first part(M2M terminals or M2M platform) which detects the communication failure sends a LOGOUT the known to the IP packet, and through the LOGOUT message communication failure status word report. After LOGOUT sent by the requesting party, the requester after receiving LOGOUT_ACK or receive timeout, release the connection. If the LOGOUT message or LOGOUT_ACK lost in the transmission of message, the requester after receiving LOGOUT_ACK timeout, release the connection, while the response can also be used in the absence of data packets after a period of time to detect the connection is invalid. Then M2M terminal can according to their own need to decide whether to launch WMMP connection again. 


\section{PROGRAMMing REALIZATION}

\section{A. Message structure}

WMMP package is made up of message header and message body, the header is required and the body is optional according to different system service.

WMMP header describes the basic information of every WMMP message. The length of it is fixed and the format is also fixed. The data elements include the following part in order: total length, message id, sequence, version, safety flag, reserved and terminal id.

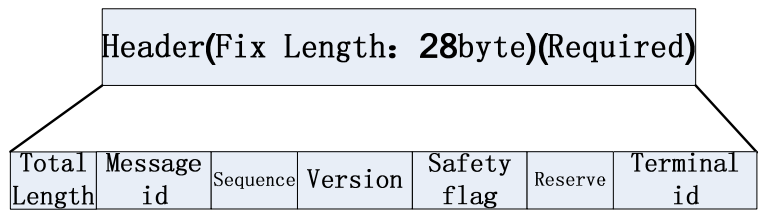

Figure 2. Data element of WMMP header

1) Total length: the total length of the whole WMMP message;

2) Message id: identify command to execute or response monitoring management, such as: remote configuration, download, update, data acquisition, namely the Command ID;

3) Sequence: a unique identifier which is produced by sender. The receiver replys the request and keeps it unchanged. The sender maintains their respective sequence (from 0X00000000 to OXFFFFFFFF).Once sending a request, the sequence of the next message increases automaticly. If the squence reaches OXFFFFFFFF, it will go back to 0x00000000.

4) Version: the version field is used to indicate the protocal version of sender. Receicer can accept or refuse the message according to its version;

5) Safety flag: this is relative to safety machanism of WMMP;

6) Reserve: reserve element has two purpose, one is keeping byte alignment, another is expending for further purpose;

7) Terminal id: this is the unique identity of every M2M terminal. It is produced by M2M platform and its validity is maintained by M2M platform too.

WMMP body is the loader of message information. The format of it is called TLV.

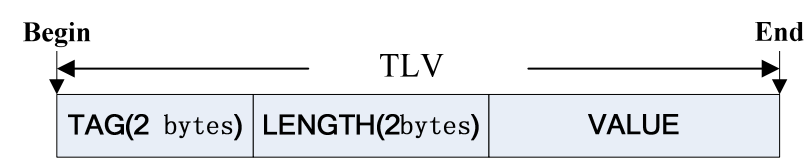

Figure 3. Data element of WMMP body

All parameters are divided into different groups and each is marked by a tag. For example, the tag of signal intensity is defined as 0x0002 and if a TLV is gotten which tag equals to 0x0002, we can know the current signal intensity easily.

\section{B. Operation process}

The normal operation process is shown in figure 4 . When the M2M terminal (here terminal is our integrated health tester) is powered on, it will send LOGIN message at first. M2M platform will send CONFIG_SET message if the M2M Terminal needs to configure. TRANSPARENT_DATA message and HEART_BEAT message will send alternately. Finally, if the operation is end and we want to quit, a LOGOUT message is sent.

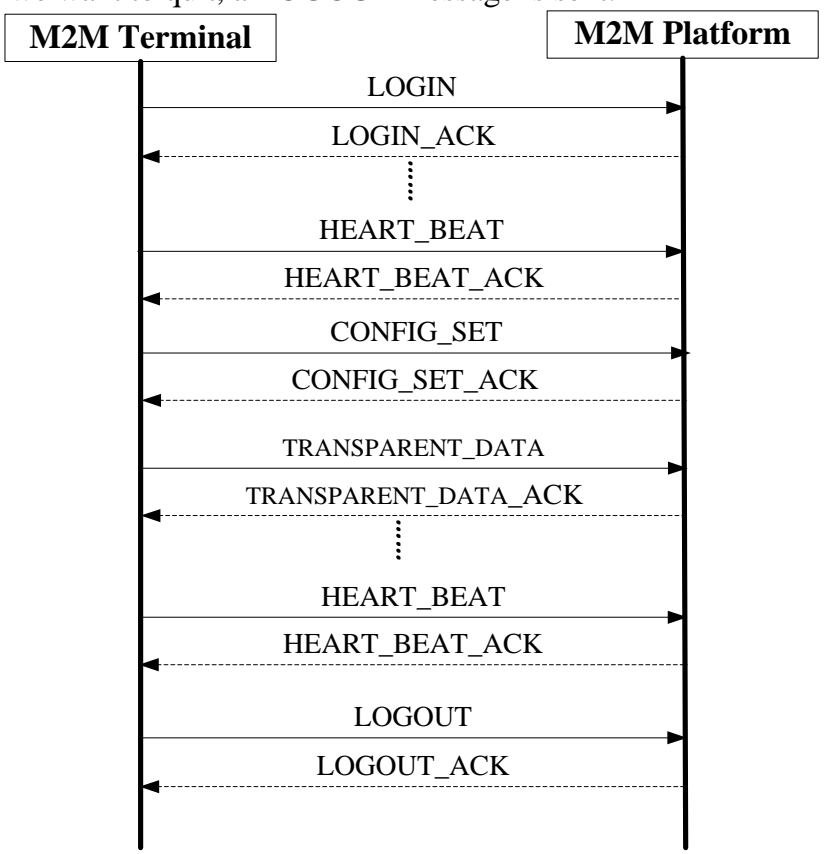

Figure 4. A normal operation process

\section{ACKNOWLEDGEMENT}

This work was supported by National High Technology Research and Development Program of China (863) (SS2012AA022805)

\section{REFERENCES}

[1] Zhu Hao, Liu Rongduo, "Progess in M2M Standards at Home and Abroad,” TELECOMMUNICATIONS NETWORK TECHNOLOGY. Beijing, vol. 10, pp. 36-39, 2010.

[2] Gao Tong, Zhu Jiajia, Luo Shengmei and Sun Zhixin "Research on M2M Functional Architecture and Security," COMPUTER TECHNOLOGY AND DEVELOPMENT. Beijing, vol. 1, pp. 250254, 2012.

[3] Chen Hongsong, Fu Zhongchuan, Zhang Dongyan "Security and trust research in M2M system,” 2011 IEEE International Conference on Vehicular Electronics and Safety. pp. 286-288, 2011.

[4] Ma Miao, "How to Realize Intelligence Health Care Under the Internet of Things Mode,” Journal of Medical Informatics. 2010.

[5] Bonacina, Stefanc, Marceglia "Towards Home Healthcare Informatics,” Proceedings 2010 2nd Conference on Human System Interactions. Beijing, vol. 33, pp. 8-11, 2012

[6] Wireless Machine-to-Machine Protocol, China Mobile Communication Corp. 
